CORPUS $\begin{aligned} & \text { Corpus } \\ & 12 \mid 2013 \\ & \text { Dialectologie : corpus, atlas, analyses }\end{aligned}$

\title{
Parole e testi : l'esperienza di un atlante
}

\section{Sabina Canobbio}

\section{(2) OpenEdition}

Journals

\section{Electronic version}

URL: http://journals.openedition.org/corpus/2302

DOI: 10.4000/corpus.2302

ISSN: $1765-3126$

\section{Publisher}

Bases; corpus et langage - UMR 6039

\section{Printed version}

Date of publication: 1 January 2013

Number of pages: $39-60$

ISSN: 1638-9808

\section{Electronic reference}

Sabina Canobbio, «Parole e testi : l'esperienza di un atlante », Corpus [Online], 12 | 2013, Online since 14 May 2014, connection on 08 September 2020. URL : http://journals.openedition.org/corpus/2302 ; DOI : https://doi.org/10.4000/corpus.2302 


\title{
Parole e testi : l'esperienza di un atlante
}

\author{
Sabina CANOBBIO \\ Università di Torino
}

Colgo volentieri l'opportunità offerta da questo numero della rivista Corpus, in cui si dà voce a esperienze di ricerca che si sono confrontate con insiemi complessi di dati, per proporre alcune riflessioni nate nel cantiere di lavoro di una impresa subregionale italiana, l'Atlante Linguistico ed Etnografico del Piemonte occidentale - ALEPO. Sarà questa l'occasione per richiamare brevemente le caratteristiche di questo atlante che ha iniziato ormai da un decennio le pubblicazioni e per fare un bilancio delle sue prime realizzazioni in rapporto non solo alle finalità per cui il progetto è nato ma anche a certi aspetti del suo corpus di materiali.

\section{Il progetto e i suoi sviluppi}

Il progetto per l'ALEPO nasce nei primi anni '80 dalla collaborazione tra la Regione Piemonte e 1'Università di Torino, con l'intento di documentare le sopravvivenze galloromanze, francoprovenzali e occitane, nelle vallate del Piemonte e per completare dunque anche sul versante italiano delle Alpi la ricerca degli atlanti regionali francesi ${ }^{1}$ che si erano sostanzialmente arrestati ai confini di stato. Indubbio dunque il suo legame, con questi atlanti, ma altrettanto evidenti rispetto ad essi numerose innovazioni prodotte dal maturare della riflessione teorica e metodologica ${ }^{2}$ e dalla stessa maggior complessità repertoriale della nostra area $^{3}$ rispetto a quella transalpina, e

1 In particolare dell'ALJA e dell'ALP.

2 In conformità anche, nel nostro caso, ad alcune linee di sviluppo proprie della « scuola torinese » di matrice terraciniana.

3 In cui le parlate occitane e francoprovenzali si incontrano con il piemontese (non solo con quello di koinè, modellato sul torinese, ma con le sue

Corpus $n^{\circ} 12$ « Dialectologie : corpus, atlas, analyses » (2013), 


\section{S. CANOBBIO}

inoltre dalle nuove possibilità di trattamento e di presentazione dei dati offerte dalle tecnologie informatiche ${ }^{4}$.

Quando si apre il cantiere di lavoro per questa nuova impresa atlantistica, l'auspicio è che essa possa in prospettiva proporre strumenti di lettura aggiornati di un territorio cruciale, cerniera di collegamento tra area linguisticamente galloromanza e area galloitalica, ma poi interessato sempre più compiutamente, tra i diversi aspetti del suo cambiamento, dall'affermarsi della lingua nazionale. Un'area insomma della cui fisionomia linguistica e culturale non possono più dare sufficiente conto le pur straordinarie documentazioni, raccolte però in una diversa dimensione di ricerca e in anni ormai troppo lontani, dell' AIS (Sprach- und Sachatlas Italiens und der Südschweiz) e dell'ALI (Atlante Linguistico Italiano).

Pur essendo l'impianto dell'ALEPO quello di un atlante, come si dice, "di scavo », destinato a documentare attraverso le parole locali i diversi aspetti della vita e del lavoro tradizionali delle comunità del Piemonte montano ${ }^{5}$, in buona parte ormai obsoleti, è chiaro che i materiali raccolti non potranno che essere il riflesso delle profonde trasformazioni sociali, culturali e linguistiche che hanno, in particolare dal secondo Dopoguerra, interessato anche questo territorio.

Entro i primi anni ' 90 vengono completate e trascritte le inchieste sul campo ${ }^{6}$, comprensive di rilievi etnofotografici ${ }^{7}$ e

diverse varietà locali) e in alcune località con il francese oltre che con l'italiano.

4 Per tutti gli aspetti di metodo e per una compiuta presentazione del progetto e del suo percorso di sviluppo si rimanda a ALEPO/Presentazione, cioè al volume che nel 2003 ha avviato le pubblicazioni dell'atlante.

$5 \mathrm{Da}$ qui la scelta come strumento di inchiesta di un questionario di tipo enciclopedico tarato su questo tipo di realtà geoantropica, il Questionnaire pour enquêtes en Pays alpin di Gaston Tuaillon, tradotto in italiano e adattato all'ambiente cisalpino da Tullio Telmon. Un repertorio ricco di circa 6000 domande, organizzate in una struttura a griglia che, alternando quesiti chiusi a quesiti aperti, favorisce tra raccoglitore e informatore lo sviluppo di quella che si chiama una « conversazione guidata ».

6 In 42 località, una almeno per ciascuna delle vallate riconosciute come galloromanze dalla letteratura scientifica; in alcune vallate particolarmente estese o linguisticamente complesse le inchieste sono state più numerose ; sono stati indagati inoltre alcuni punti «fuori territorio » sia 
l'équipe di lavoro si trova di fronte alle delicate scelte connesse con l'archiviazione e il trattamento di un corpus di materiali non solo di ingentissime proporzioni ma anche, ancor più di quanto fosse previsto, complesso e articolato (spesso più risposte per ciascun punto, illustrazioni, etnotesti, informazioni pragmatiche e metalinguistiche, annotazioni di vario tipo, ecc.). Non a caso in quegli stessi anni viene avviata nel cantiere dell'ALEPO la prima sperimentazione dell'informatizzazione dei dati ; una svolta che si rivelerà assai fruttuosa, anche se non certo indolore per le difficoltà di riconvertire alle regole rigide dello strumento informatico materiali raccolti, e sottoposti alle elaborazioni preliminari, nella prospettiva di archiviazione e sbocchi editoriali del tutto tradizionali e «cartacei». Grazie a questo impegnativo processo ${ }^{8}$ l'ALEPO si è dotata della ben

in senso linguistico sia in senso culturale, località cioè linguisticamente galloitaliche e culturalmente pianigiane. L'impresa ha visto l'impiego di più raccoglitori, per la cui scelta è stata privilegiata, quando possibile, l'appartenenza alle comunità indagate (su questo aspetto, oltre che a ALEPO / Presentazione, si rimanda a Regis 2001 che ne rileva l'importanza nelle dinamiche di inchiesta). Per quanto riguarda gli informatori (hanno collaborato alla ricerca più di 200 parlanti nativi), la loro scelta non si è basata su criteri di rappresentatività sociolinguistica ma sulla competenza linguistica e culturale rispetto alle caratteristiche dell'area. I rilievi sono stati integralmente registrati su supporto analogico (l'archivio sonoro, poi riprodotto su supporto digitale, assomma a circa 1300 ore). I materiali raccolti sono stati trascritti dagli stessi raccoglitori in grafia fonetica, originariamente secondo una versione semplificata del sistema ALF-Rousselot proprio della tradizione geolinguistica francese, in seguito però poi sostituita per le pubblicazione dell'atlante dal sistema IPA. La trascrizione non ha interessato solo le risposte puntuali e l'articolarsi più o meno complesso delle testimonianze in etnotesti (sui quali ritorneremo dopo), ma anche le loro componenti pragmatiche, paralinguistiche e metalinguistiche (gesti, risate, esitazioni, ripensamenti, autocorrezioni, pause, osservazioni degli informatori).

7 I rilievi fotografici sono stati poi quasi sempre ripresi e completati da alcuni collaboratori specializzati. L'ALEPO dispone a tutt'oggi di circa 3000 schede etnofotografiche (relative ad attrezzi, strutture abitative, masserizie) che costituiscono oggi un prezioso collegamento tra i referenti oggettuali indagati e le parole che, secondo le testimonianze dei parlanti locali, li significano.

8 Tuttora in divenire, non solo per i normali motivi di manutenzione e di aggiornamento del software, ma perché i problemi posti dal trattamento 


\section{S. CANOBBIO}

articolata struttura di una banca dati, funzionale alle esigenze di archiviazione dei suoi complessi dati, e anche del software in grado di farne uscire (in buona parte automaticamente) oltre alle tavole dell'atlante, i materiali complementari e gli apparati.

Alla pubblicazione, nel 2003, di un volume di presentazione della ricerca e dei suoi sviluppi hanno fatto seguito (tra il 2004 e il 2007) quelle dei tre moduli del I Volume dell'atlante, tutto dedicato al Mondo vegetale (I-I. Alberi e arbusti ; I-II Erbacee; I-III Funghi e licheni), completato nel 2008 dall'Indice dei tipi lessicali e da una prima proposta di consultazione interattiva dei dati.

Dell'inizio del 2013 è l'edizione del Volume III, dedicato al Mondo animale (III-I Fauna ; III-II Caccia e pesca), che si è deciso di pubblicare di seguito al I, forzando l'ordine previsto dal Piano editoriale, per completare in questo modo la presentazione dei materiali raccolti dall'ALEPO attorno alla fitonimia e alla zoonimia popolari. Queste ultime costituiscono, infatti, settori del lessico dalle caratteristiche peculiari, espressione di saperi naturalistici e linguistici complessi e profondamente radicati nelle culture locali che appaiono però oggi in una fase particolarmente critica di destrutturazione e presentano dunque analoghi problemi teorici e metodologici, sia nel trattamento dei dati sia nella loro lettura.

Attualmente sono in fase di preedizione i materiali per il Volume V, dedicato a Lo spazio e il tempo.

Quali sono le caratteristiche di questo oggetto, che continuiamo a chiamare atlante linguistico, ma che indubbiamente è qualche cosa di ben diverso da quei modelli da cui eravamo partiti, dando così ragione del percorso compiuto nel cantiere dell'ALEPO nel suo ormai trentennio di attività?? Ogni volume,

di alcuni tipi di dati non sono stati, come vedremo, ancora compiutamente affrontati.

9 Indubbiamente uno degli aspetti più critici, e più criticati, delle ricerche per gli atlanti, e anche dell'ALEPO, è proprio il tempo che essi impiegano, per la complessità della loro realizzazione, a rendere disponibili alla consultazione i dati raccolti. 
cui fa capo un numero variabile di moduli ${ }^{10}$, consta in primo luogo di una componente cartacea, che presenta però solo una selezione delle voci generate dalla redazione dei materiali. Queste ultime sono formate da tavole (cioè dalle carte linguistiche vere e proprie ${ }^{11}$, sulle quali sono riportate per ogni punto solo le risposte individuate come principali) e dai tabulati dei materiali integrali di quelle stesse voci (risposte puntuali, etnotesti, apparati di note), preceduti da una nota redazionale. Questa componente cartacea è accompagnata da un CD-Rom, che presenta in forma di libro elettronico ${ }^{12}$ il complesso di tutte le voci del modulo, oltre agli indici e agli altri strumenti di accesso ai dati. A conclusione del I volume, nel CD-Rom allegato ad ALEPO I/Indice, è stata proposta, in via sperimentale, una modalità più interattiva di lettura delle carte ${ }^{13}$, con la possibilità di ricerche incrociate ed elaborazioni areali dei dati, sulla base della tipizzazione delle forme linguistiche ${ }^{14}$.

Questo modello di atlante, in parte cartaceo e in parte elettronico, sperimentato nei volumi I e III, viene incontro a una delle principali preoccupazioni dell'ALEPO, quella cioè di non perdere nulla della complessa ricchezza che caratterizza il corpus dei dati raccolti nelle sue inchieste. Esso tenta nei fatti di superare almeno in parte, grazie alla natura composita della voce, gli angusti limiti della carta linguistica tradizionale, sulla quale è ben difficile, senza comprometterne la leggibilità, non

10 Tre ad esempio, come si è visto, nel I volume (e oggetto ciascuno per la loro entità, di pubblicazioni autonome); due nel III volume (pubblicati insieme).

11 Come già più volte osservato, cfr. ad esempio Canobbio 2003, è evidente la difficoltà di descrivere i nostri nuovi atlanti con la metalingua tradizionale della geolinguistica.

12 Da sfogliare in modo molto simile a un libro normale con qualche possibilità interattiva; si possono, ad esempio, ingrandire le carte o i dettagli dei materiali di complemento, si possono stampare le voci che interessano, anche in formato A4 e in bianco e nero, ecc.

13 Uno sviluppo ne è attualmente in progettazione anche per i materiali del III volume, forse (è una delle ipotesi allo studio) da rendere disponibile in rete.

14 Un passaggio questo molto delicato che ha imposto scelte cruciali di cui si dà ampio conto in Cerruti, Regis 2008. 


\section{S. CANOBBIO}

solo riportare in corrispondenza di ciascuno dei punti indagati più di un dato, ma anche accompagnarlo con l'insieme delle informazioni che lo contestualizzano e lo qualificano. Le voci dell'ALEPO infatti non solo accostano immediatamente alla rappresentazione cartografica delle risposte principali il listato sintetico di tutte le altre risposte, ma poi le pagine dei materiali propongono in forma tabulare ${ }^{15}$ l'insieme integrale e completo dei contenuti, primari e di complemento, archiviati nella banca dati e correlati con quella voce, compresi gli etnotesti e le loro traduzioni italiane. Il corredo informativo che accompagna ogni dato raccolto comprende in primo luogo la stringa identificativa dell'informatore che, oltre a collegarlo con la località di inchiesta tramite il relativo codice, dà indicazione del suo sesso, grado di scolarità, anno di nascita ${ }^{16}$, permettendo così di collegare immediatamente il dato stesso ad alcuni parametri fondamentali per la sua valutazione.

L'apparato di note prevede le due serie distinte di quelle prodotte da interventi degli informatori (oltre che dalle specificazioni di tipo pragmatico, che rimandano a gesti, esitazioni, ecc.) e di quelle prodotte dalla redazione. Proprio la ricchezza delle note che raccolgono le osservazioni dei parlanti permette, insieme alla disponibilità dei loro dati personali, di rilevare non di rado, anche se non specificamente indagati, anche aspetti sociolinguistici e percettivi.

Una delle soluzioni che si sono rivelate più fruttuose per la salvaguardia e la valorizzazione del patrimonio documentario emerso dalle inchieste è quella del cosiddetto "Questionario a posteriori ", che permette di recuperare (codificandoli e inserendoli nell'archivio, dunque rendendoli disponibili alla consultazione), dati emersi non dalle domande del questionario

15 La struttura di questa parte delle voci si può cogliere, per quanto parzialmente, nei particolari di alcune voci riportati nelle Figure allegate. Il formato di questa pubblicazione sconsiglia evidentemente qui la riproduzione integrale di carte e/o di pagine dei materiali dell'atlante che risulterebbero non facilmente leggibili.

$16 \mathrm{Si}$ veda la terza colonna da sinistra nei particolare dei materiali di alcune voci, riportati nelle Figure allegate. 
bensì da interventi spontanei degli informatori ${ }^{17}$. Questa modalità di trattamento e di archiviazione si è rivelata preziosa proprio nel trattamento dei materiali relativi alla flora e alla fauna, dal momento che una parte non indifferente dei relativi etnosaperi è, come ricorderemo ancora qui sotto, destinata a sfuggire alla pur minuta griglia di qualunque questionario. In molti casi dati recuperati in questo modo costituiscono ora nelle relative voci importanti tasselli informativo per la lettura del quadro areale o come integrazioni di tipo etnoculturale ${ }^{18}$.

\section{Primo bilancio}

Qual è dunque un provvisorio bilancio che si può fare dell'impresa sulla base di queste sue prime realizzazioni, in rapporto alle aspettative generali ma anche alla specificità degli argomenti fino ad ora trattati?

L'ALEPO ha scelto, come abbiamo visto, per mettere alla prova i propri materiali e il proprio metodo due settori del lessico popolare assai scivolosi per gli atlanti linguistici, quello fitonimico e quello zoonimico. Che sono tali principalmente per la difficoltà, messa bene in luce dalle acquisizioni dell'etnolinguistica ${ }^{19}$, di ricostruire tramite le domande di un questionario, e dunque quasi inevitabilmente sulla base delle categorie previste dalle classificazioni scientifiche, l'effettivo patrimonio delle denominazioni popolari e la trama di rapporti su cui esse si basano.

$17 \mathrm{Si}$ veda a questo proposito (e più in generale sull'organizzazione dei dati nella Banca dati) Raimondi 2003.

18 Come nel caso della carta supplementare relativa a un prodotto ricavato dalle bacche di ginepro di cui si dice qui sotto al punto 2 .

19 La letteratura specifica a questo riguardo ormai talmente ampia che non se ne può dare qui che qualche minimo cenno, a partire dalla riflessione maturata nel cantiere dell'ALEPO ; si vedano in particolare Calleri 1990, Canobbio 1999, 2004, 2005, 2007, 2012a, 2012b ; Calleri, Canobbio, Telmon 2001 ; Canobbio, Raimondi 2004 e le rispettive bibliografie. Tra i contributi più recenti alla speculazione collettiva su queste tematiche si rimanda a Ortalli, Sanga 2004 ; Mendicino, Prantera, Maddalon 2004 ; Prantera, Mendicino, Citraro 2010. Doveroso infine segnalare ancora una volta la rivista Quaderni di semantica, come sede di pubblicazione negli ultimi anni di moltissimi lavori che hanno ben messo in luce questi aspetti. 


\section{S. CANOBBIO}

A questo problema di fondo è venuto negli ultimi decenni sempre più decisamente ad aggiungersi, complicando ulteriormente l'elicitazione dei dati, l'impoverimento delle competenze specifiche, sia linguistiche sia culturali, dei parlanti, anche di quelli delle nostre vallate alpine.

Questi diversi ordini di difficoltà emergono indubbiamente dalle voci dei due primi volumi dell'ALEPO e sono messi in evidenza dalle note di presentazione che illustrano e valutano, modulo per modulo e voce per voce ${ }^{20}$, la produttività delle domande del questionario, la qualità dei dati raccolti, le difficoltà specifiche incontrate nel loro trattamento, le loro valenze culturali.

Indubbiamente molte delle voci (e delle carte) di tutti i moduli appaiono lacunose e la simbologia che dà conto della motivazione dell'assenza di risposta riproduce sinteticamente i molti " forse ", " non so ", " conosco ma non dire un nome », «non ricordo» con cui si è manifestata nel corso dei rilievi l'attuale, generalizzata insicurezza da parte dei nostri informatori attorno a saperi e a settori della lingua ormai marginali nella vita delle loro comunità ${ }^{21}$. L'insicurezza del sapere si incrocia insomma nelle testimonianze con quella del saper (ancora) dire.

Anche dove le risposte ci sono, non di rado esse sono accompagnate da note che segnalano dubbi redazionali sulla loro «attendibilità ». Però a rassicurarci sull'opportunità della scelta di riportare comunque il complesso delle risposte delle nostre fonti, anche nelle sue componenti più critiche e magari contraddittorie, è sempre utile ricordare ${ }^{22}$ le parole scritte da Vittorio Bertoldi in rapporto alla ricerca fitonimica, cui il grande studioso ha dedicato studi fondamentali, ma valide naturalmente anche a proposito dei nomi di animali :

20 Cfr. per le note introduttive dei moduli Canobbio 2004, 2005, 2007, 2013 ; inoltre le note redazioni premesse a ogni voce.

21 Anche se gli ultimi anni hanno indubbiamente visto anche nelle nostre vallate un riaccendersi di attenzione di tipo naturalistico (ad esempio in rapporto alla medicina tradizionale) e lo svilupparsi di iniziative di recupero perché la montagna «torni a vivere » (cfr. per lacune di queste esperienzeVarotto 2013), questo non corrisponde necessariamente anche a un recupero in termini di specificità linguistica locale.

22 Ancora una volta; cfr. Canobbio2005 : 57. 
Parole e testi

Ho detto tutto questo per dare un'idea delle difficoltà che incontrerebbe non tanto il botanico, ma lo stesso linguista che volesse fare distinzioni troppo rigide del suo materiale e propendesse troppo sovente agli ostracismi di nomi ritenuti d'origine sospetta. Quello che il nostro empirismo retrospettivo chiama voce popolare e voce letteraria come, del resto, anche quello, che esso chiama voce indigena e voce d'accatto, neologismo e voce antiquata, evoluzione normale e anormale non sono che fasi fuggitive nella storia dei linguaggi, non sono che riflessi della vita interiore dei parlanti, fatta di contrastanti tendenze ed esposta a continui influssi. Dunque nel compiere la raccolta, nessun segno di stupore, nessuna prevenzione, nessun partito preso. Tutto ci interessa quanto rispecchia fedelmente la realtà linguistica di quel dato momento e in quel dato luogo, anche quello che ad altri può sembrare artificioso, improbabile, accidentale, poiché tutto appartiene a una materia in perenne formazione ed evoluzione, la quale s'arresta e s'irrigidisce appena quand'è giunta nei nostri libri. (1927 : 30).

Parole che (a giustificare questa lunga citazione) sembrano ancora attualissime, come è confermato da molte altre riflessioni maturate negli ultimi decenni a proposito delle attribuzioni di veridicità e/o di falsità a etichette denominative che hanno per molti motivi, come ha scritto Jean-Philippe Dalbera un carattere « del tutto fluido» (2002: 836-838), e inoltre motivazioni profonde che sfuggono spesso alla nostra attuale capacità interpretativa $^{23}$. Basti pensare all'esempio offerto dalle denominazioni dei rapaci notturni che sembrano mostrare, nelle voci dell'ALEPO ${ }^{24}$ come del resto negli altri atlanti e repertori, con il

23 Un ulteriore invito alla prudenza nella valutazione di dati apparentemente « inspiegabili » che ci viene dalle preziose sintesi romanze che ai nomi di molte animali sta dedicando l'ALiR.

24 Cfr. per ALEPO III.I tre voci principali, 147 Civetta (Athene noctua), 149 Gufo comune(Asio otus), 153 Barbagianni (Tyto alba) e alcune altre in cui sono confluiti dati attribuibili ad altri strigidi : 145/s Allocco (Strix aluco), 146/s Assiolo (Otus scops), 151 Gufo di palude (Asio flammeus), 152/s Gufo reale (Bubo bubo). 


\section{S. CANOBBIO}

loro continuo sovrapporsi e scambiarsi di posto in rapporto ai referenti, un quadro caotico e apparentemente privo di logica e ragionevolezza per lo studioso che deve ordinarli e studiarli ${ }^{25}$.

In ogni caso il contributo che i materiali dall'ALEPO, nonostante i loro aspetti problematici, possono portare a una migliore conoscenza della fisionomia plurilingue del Piemonte occidentale nelle sue complesse dinamiche in continua evoluzione, appare da queste prime uscite promettente. Anzi nelle parti del lessico sin qui trattate rimaste, proprio per la loro marginalità, relativamente più al riparo di altre nel processo di italianizzazione delle parlate locali, sembra potersi leggere ancora bene l'articolarsi dei rapporti tra i tre principali domini a contatto nell'area, quello francoprovenzale, quello occitanico e quello pedemontano.

Un esempio molto interessante è offerto, tra gli altri, dalla voce Nocciolo (Corylus avellana, ALEPO I-I.80), che mostra apparentemente una distinzione chiara tra le tre aree, con le forme dei lessotipi AVELLANA soprattutto nelle vallate occitane, quelle da CORYLU prevalenti nelle valli francoprovenzali e quelle da NUCEOLA a caratterizzare l'area pedemontana galloitalica. Ma come ha mostrato bene Riccardo Regis $(2006 \text { e } 2008)^{26}$ l'analisi puntuale dei dati nell'intera rete dei punti mostra un sistema in movimento in cui i lessotipi prevalenti nei domini galloromanzi appaiono in via di cedimento ${ }^{27}$ sotto la pressione del tipo pedemontano, peraltro probabilmente rinforzata dal fatto che esso è condiviso, sia pure con esiti diversi, dall'italiano. Non a caso, nota Regis, l'area in cui « sembra consumarsi l'agone più stimolante» $(2006: 157)$ tra $i$ tre lessotipi è la Valle di Susa ${ }^{28}$, in cui i domini francoproven-

$25 \mathrm{Si}$ veda a questo proposito Canobbio 2012 ; Dalbera 2006 (in particolare alle pp. 275-338, dedicato appunto a queste specie e ai loro nomi); Evans 1960.

26 Cfr. anche Cerruti, Regis 2007 ; Canobbio, Raimondi 2004.

27 Per ora più vistosi, come prevedibile, nel nome del frutto (che rappresenta un prodotto pregiato oggetto di commercio per varie zone del Piemeonte) che in quello della pianta.

28 I materiali pubblicati hanno già contribuito in realtà anche ad alimentare il vivace dibattito in corso per una migliore definizione delle caratteristiche linguistiche di un'altra parte molto delicata del territorio interes- 
zale e occitanico si incernierano tra loro, ma in cui anche il piemontese, soprattutto nel fondovalle, si è addentrato più precocemente che altrove ${ }^{29}$.

In molte voci, in molte carte, a emergere è una perdurante ricchezza di lessotipi, la cui sopravvivenza forse non ci si sarebbe attesi nell'attuale indebolimento delle parlate locali. Un caso molto significativo in questo senso è ad esempio quello del vivissimo polimorfismo lessicale che emerge dalla voce Narciso (ALEPO I-II.8, narciso selvatico-Narcissus poeticus), riconducibile addirittura a 16 tipi $^{30}$, alcuni motivati dalla morfologia della pianta ma più frequentemente nati da processi culturali.

Di non minore interesse sono peraltro, nell'economia dell'opera, anche le voci caratterizzate dalla presenza di un solo tipo lessicale (ad esempio quella dedicata al Lupo, ALEPO III-I 267 lupo-Canis lupus), ma di contro ricchissime di testimonianze su tradizioni e storie locali.

In qualche caso il fatto che l'attestazione, magari isolata, di una denominazione sia accompagnata da una spiegazione di valenza etnografica offre una traccia preziosa per ipotesi interpretative altrimenti impraticabili. Penso ad esempio al nome dame d'aigo attestato nel P. 630 Monterosso Grana (in alternativa a cavalot) per l'Opilionide (ALEPO III-I.51 opilioninePhalangium opilium). Come già osservato (Canobbio 2003 : 5152) la spiegazione viene dal collegamento di questo nome (« dammi dell'acqua ») a una filastrocca che si recitava, tenendolo fermo per una delle lunghe zampe, perché il falangio emettesse una goccia d'acqua dall'addome : cavalin, cavalot, dame d'aigo snu ses mort («cavallino, cavalot, dammi dell'acqua altrimenti sei morto »).

sato dalla ricerca, cioè la sua sezione più meridionale, in cui accanto a tratti pedemontani e occitanici si fanno evidenti, e in alcuni casi forse preponderanti, quelli di tipo ligure (cfr. ad esempio alcuni contributi nel recente Duberti, Miola 2012).

$29 \mathrm{Si}$ veda a questo proposito nella Fig. 1 il particolare che mostra i dati di una parte di questo territorio nei materiali della Voce I.I.60.

30 Cfr. Calleri, Canobbio, Telmon 2002 :95-116 ; e la nota redazionale della voce, firmata da T. Telmon. 


\section{S. CANOBBIO}

Spesso le osservazioni che accompagnano i nomi attestati per una specie ribadiscono con convinzione, nonostante il riscontro contrario della realtà, le credenze che li motivano, com'è nel caso del povero Orbettino (ALEPO III-I 320 orbettino-Anguis fragilis), che voci e detti popolari vogliono cieco, o addirittura privo di occhi, oltre che pericolosissimo ${ }^{31}$.

E' evidente l'interesse delle voci che risultano collegate tra di loro da diversi tipi di legami e i cui significati linguistici e culturali dunque si completano nel confronto, come è nel caso di quelle dedicate a specie arboree e arbustive e ai loro frutti. Così è, ad esempio, per le voci dedicate al Ginepro (ALEPO I-I 85 ginepro-Juniperus communis) ${ }^{32}$ e alle sue bacche (ALEPO II 86 bacche di ginepro) ma anche a una particolare composta da esse ricavato in alcune località della Val di Susa (ALEPO I-I 86/s composta di bacche di ginepro). È quest'ultimo uno di quei casi in cui alle voci prodotte da domande del questionario se ne è aggiunta un'altra, supplementare, generata dalle attestazioni spontanee di alcuni informatori. In essa si è potuto segnalare una specificità lessicale e culturale che non avrebbe avuto adeguato rilievo (e non sarebbe stata indicizzata) se semplicemente inserita nelle note di un'altra voce ; in questo caso specifico, confusa tra i numerosi impieghi, officinali e alimentari, delle bacche del ginepro.

\section{Gli Etnotesti}

Abbiamo visto che oltre che con dati puntuali, con parole, l'ALEPO si trova a confrontarsi con un tipo di materiale meno usuale nei corpora degli atlanti e meno funzionale alla loro struttura, cioè con dei testi, anzi con quelli che abbiamo già prima citato come etnotesti ${ }^{33}$ Essi hanno avuto e hanno, per questa ricerca un ruolo determinante sia come strumento eu-

31 Si veda un particolare dei materiali della voce ALEPO nella Fig. 3 ; per i nomi dell'orbettino e per le credenze relative cfr. Canobbio 2003.

$32 \mathrm{Si}$ veda un piccolo particolare dei materiali della voce nella Fig. 2.

33 Per l'impiego degli etnotesti nell'ALEPO cfr. Canobbio 1985, 1989, 2011 ; per le esperienze francesi da cui la ricerca per l'ALEPO lo ha mediato, cfr. almeno Bouvier et Alii 1980, Bouvier 1992. 
ristico nelle fasi di inchiesta sia poi come componente preziosa e qualificante, ma anche cruciale, del suo corpus di dati.

Alcune scelte fatte fin dall'inizio, sono risultate accorte, a partire da quella di trattare come etnotest ${ }^{34}$ tutti quei materiali che, nel corso dei rilievi non siano stati ottenuti come traduzioni da parola a parola ma che rappresentino l'espressione autonoma della cultura dei parlanti (testimonianze di usi e tradizioni ; descrizioni di oggetti e pratiche ; forme brevi quali proverbi, detti, formulette e filastrocche; storie ; opinioni e valutazioni anche metalinguistiche, ecc.). Ma anche quella di conservare integralmente le interazioni raccoglitore-informatore, riconoscendo dunque esplicitamente il ruolo della situazione-inchiesta nella genesi degli etnotesti. Collegata in buona misura a questa la scelta inoltre, maturata in seguito, di restituire nelle trascrizioni il discorso orale delle nostre fonti nel suo complesso multilinguismo, compresi i molti segmenti in italiano ${ }^{35}$. Proprio le ragioni pragmatiche e le modalità dell'alternarsi nelle testimonianze dei diversi codici - italiano ${ }^{36}$, piemontese, parlata locale - sembrano poter costituire una delle più interessanti piste future di lettura degli etnotesti dell'ALEPO (Regis 2001). Soprattutto mettendoli in rapporto con le risposte alle domande puntuali che, come più volte notato, sottopongono il parlante a un più serrato autocontrollo nell'uso delle componenti del suo repertorio rispetto a quanto avviene nel discorso libero.

Altre scelte fondamentali e qualificanti dovranno però ancora essere fatte perché le potenzialità di questa parte del corpus dell'ALEPO prendano forma. La redazione sta in realtà ancora cercando soluzioni convincenti al problema, solo apparentemente tecnico, di inserire al meglio queste centinaia di testi orali nella struttura dell'atlante, sfruttandone appieno le molteplici valenze e mettendone in luce tutti gli specifici significati, non solo genericamente linguistici, ma etnolinguistici, sociolinguistici, testuali. Un problema che nel I e nel III volume è

34 Canobbio $1985: 210$.

35 Come si vede ad esempio nella Fig. 3, al P : 380 Bardonecchia.

36 Quando verranno editati materiali etnotestuali più numerosi ed estesi, essi potranno costituire indubbiamente una sede importante di analisi dell'italiano regionale di questa parte del Piemonte. 


\section{S. CANOBBIO}

stato affrontato in modo solo parziale e provvisorio collocando gli etnotesti, anche perché il loro numero e le loro dimensioni lo permettevano, in uno spazio dedicato tra i materiali che accompagnano le carte ${ }^{37}$. È però evidente che in questo modo gli etnotesti vengono presentati solo come contesti dei dati puntuali, ed eventualmente come fonte di ulteriori informazioni, senza alcuna possibilità di analizzarli, annotarli, indicizzarli nella loro specificità. Questo modello non può evidentemente che apparire inadeguato in rapporto al numero e all'estensione di tutti quegli altri etnotesti prodotti, ad esempio, attorno ai temi del lavoro agricolo e artigiano, agli usi sociali e alle tradizioni delle comunità. La complessa ricchezza dei loro significati meriterà e richiederà una lettura più puntuale e più analitica.

Le soluzioni allo studio a questo proposito ${ }^{38}$ prevedono per gli etnotesti interventi quale l'introduzione di un sistema di trascrizione fono-ortografica, per facilitarne la lettura anche da parte di utenti non specialisti, e inoltre di notazioni di tipo conversazionale che mettano meglio in luce la loro natura di «testi orali ». Ma anche, nella prospettiva di un più esteso impiego del supporto informatico, lo sviluppo di applicazioni di tipo ipertestuale, che possano interconnettere efficacemente, e immediatamente, etnotesti, dati cartografati, apparati di note, iconografia. Sembra necessario comunque un cambio di prospettiva, che porti al superamento dell'attuale presentazione degli etnotesti come meri supporti informativi ed esplicativi e permetta, grazie a un adeguato trattamento (in primo luogo la tokenizzazione dei testi stessi) di analizzarli compiutamente nelle loro diverse componenti e sui diversi piani linguistici e culturali.

Già fin d'ora, comunque, gli etnotesti si mostrano luogo privilegiato di osservazione non solo di fatti linguistici ma dei molti processi dialettici in evoluzione che caratterizzano il territorio interessato dalla nostra ricerca ${ }^{39}$. Un territorio, per una buona parte montano, assai diversificato al suo interno, non solo

37 Come si può notare dagli esempi delle Figure allegate.

38 Cfr. a questo proposito Baratto 2011.

39 Cfr. Canobbio 2001. 
da valle a valle, ma da paese a paese perché così lo hanno disegnato vicende storiche secolari e poi sviluppi socioeconomici e culturali che hanno interessato proprio le generazioni da noi incontrate nelle nostre inchieste. Si pensi, ad esempio, all'incidenza di fatti dirompenti quale lo spopolamento che, smembrando e disperdendo i gruppi umani originari, ha svuotato in molti paesi di significato la stessa parola «comunità »e ha visto la sostanziale scomparsa di molte delle attività attorno a cui ruotava l'economia locale. Oppure la riconversione turistica, per cui molte vallate hanno subito massicciamente l'impatto di fenomeni, interessi, flussi provenienti da altrove.

Questo territorio è inoltre terra di frontiera, interessata dalla presenza di minoranze linguistiche. La coscienza identitaria dei nostri informatori, come spesso emerge dalle testimonianze, sembra tuttora molto attenta (nonostante il sempre più deciso spostamento dei centri di influenza al di fuori dalle vallate) a marcare la diversità nei confronti della pianura piemontese, e l'affinità con l'Oltralpe.

Proprio il collasso dei tradizionali assetti territoriali, umani e culturali, ha fortemente condizionato anche il rapporto tra passato e presente. Un segnale ce ne danno gli etnotesti dei volumi I e III, ad esempio con l'impiego generalizzato da parte dei parlanti dei verbi al passato (diffusissimo l'imperfetto), anche quando essi si riferiscono a oggetti, pratiche, elementi della natura che pure fanno parte ancora del presente, ma il cui significato culturale si pone evidentemente in un tempo altro. Del resto le evoluzioni socioeconomiche e/o la riconversione del territorio hanno effettivamente trasformato l'ambiente naturale e il rapporto degli uomini con esso, e non caso spesso in queste testimonianze si riferisce dei saperi tradizionali, ereditati dalla memoria comunitaria, con un atteggiamento tra il divertito e lo scettico, alla luce di altre conoscenze e di altre convinzioni nel frattempo maturate.

Gli etnotesti sono poi il luogo in cui vistosamente si manifestano anche le incrinature nella memoria della «letteratura orale » tradizionale ; lo si vede bene nelle attestazioni di 


\section{S. CANOBBIO}

forme brevi quali proverbi, detti, formulette, la cui formulazione risulta spesso ormai esitante, imprecisa e lacunosa ${ }^{40}$.

Insomma il discorso del parlante che incontriamo oggi sul campo indica sempre più spesso la natura ormai composita della sua "visione del mondo » e della sua enciclopedia, oltre che delle sue competenze, costruite attraverso la rete complessa delle sue appartenenze a diverse comunità, ristrette e allargate, reali e magari anche virtuali.

\section{Prospettive}

In definitiva, abbiamo visto che sono ancora numerose le scelte, non solo tecniche e organizzative, che dovranno maturare nei prossimi anni nel cantiere dell'ALEPO, anche in rapporto al susseguirsi di moduli e di volumi dai contenuti molto diversi. Basti pensare alle difficoltà connesse con l'inserimento nel tessuto dell'atlante del repertorio fotografico originale, che nei due volumi pubblicati non ha ancora avuto occasione di essere utilizzato che marginalmente ${ }^{41}$ ma che sarà elemento sostanziale, ad esempio, dai volumi dedicati al lavoro agricolo e all'allevamento.

Sia per l'inserimento adeguato degli etnotesti e delle fotografie sia per l'individuazione di modalità di consultazione dei dati più interattiva potranno rivelarsi risolutive, come accennato, le possibilità offerte da un maggior impiego delle tecnologie informatiche e dalla disponibilità sempre più ampia $\mathrm{e}$ incisiva dei materiali in formato elettronico. La pubblicazione direttamente in rete potrebbe rendere inoltre l'atlante più facilmente accessibile sia alla comunità scientifica sia a un pubblico allargato di lettori interessati, primi tra tutti coloro che vivono nell'area toccata dalla ricerca.

Tuttavia se l'apporto della tecnologia potrà forse farci superare, con il contributo di una adeguata équipe di collabo-

40 Come si nota nelle due attestazioni di un detto sull'orbettino presenti nella Fig. 3.

41 Alcune fotografie appartenenti al repertorio iconografico raccolto dall'ALEPO sono state introdotte (secondo modalità del tutto provvisorie e sperimentali) solo in voci del II modulo del III volume, relative a strumenti di cattura di animali. 
ratori informatici, molti dei vincoli che oggi ci condizionano, e magari anche indicarci strade nuove per interpretare i nostri dati e metterli a disposizione di più utenti, il lavoro di archiviazione e di elaborazione dei materiali non potrà che passare attraverso il lavoro minuzioso di una redazione qualificata. E certo spaventa, guardando il piano editoriale dell'opera che prevede complessivamente 9 volumi, l'entità dei dati ancora da trattare, immane rispetto alle nostre sempre sottodimensionate forze redazionali. Non va taciuto insomma che gli sviluppi dei lavori dipenderanno in una buona parte, per questa come per tutte le altre imprese collettive che richiedono strutture organizzative onerose, dal verificarsi delle condizioni per un regolare sostentamento del cantiere di lavoro e per la pubblicazione dei suoi risultati di ricerca.

Ma il rischio più grosso che l'ALEPO ${ }^{42}$ corre è quello che le sue carte e gli altri suoi materiali rimangano muti, non siano letti, interpretati, interrogati ${ }^{43}$; che nessuno cerchi di rispondere alle molte domande che essi pongono. Noi stessi che nel suo cantiere lavoriamo, oberati dalla mole di lavoro e di problemi che l'allestimento dell'atlante comporta, siamo tornati fino ad ora troppo poco a esercitare il «mestiere del geolinguista » sulle sue voci, per sfruttarne il tesoro.

\section{Références bibliographiques}

AIS = Jaberg K. \& Jud J. (1928-40), Sprach- und Sachatlas Italiens und der Südschweiz, Zofingen : Ringier u. C.

ALEPO / Questionario = Canobbio S. \& Telmon T. (éd.) (199394), Atlante Linguistico ed Etnografico del Piemonte Occidentale. Questionario, I, Introduzione; II, Testo ; III, Indice lemmatizzato. Torino : Regione Piemonte.

42 Come ogni altro atlante linguistico e forse come ogni altra impresa scientifica che allestisca e tratti corpora di dati.

43 Cfr. a questo proposito le condivisibili considerazioni di Telmon 2009 e gli interventi di Alberto Sobrero, cui egli fa ampio riferimento, a una tavola rotonda palermitana su « Il mestiere di geolinguista oggi » pubblicata in Matranga, Sottile 2007 : 2005-235. Ma medesime preoccupazioni sono state espresse anche da Jean-Philippe Dalbera, cfr. ad esempio Dalbera 2009. 


\section{S. CANOBBIO}

ALEPO $/$ Presentazione $=$ Canobbio S. \& Telmon T. (éd.) (2003), Atlante Linguistico ed Etnografico del Piemonte occidentale-ALEPO. Presentazione e guida alla lettura. Pavone Canavese : Priuli \& Verlucca Editori.

ALEPO I-I = Atlante Linguistico ed Etnografico del Piemonte occidentale - ALEPO (2005). Volume I, Il mondo vegetale, Modulo I, Alberi e arbusti, Pavone Canavese : Priuli \& Verlucca Editori [volume, carte, CD-Rom].

ALEPO I-II = Atlante Linguistico ed Etnografico del Piemonte occidentale - ALEPO. Volume I, Il mondo vegetale, Modulo II, Erbacee. (2007). Scarmagno : Priuli \& Verlucca Editori [volume, carte, CD-Rom].

ALEPO I-III = Atlante Linguistico ed Etnografico del Piemonte occidentale - ALEPO. Volume I, Il mondo vegetale, Modulo III, Funghi e licheni (2004). Pavone Canavese : Priuli \& Verlucca Editori [volume, carte, CD-Rom].

ALEPO I/Indice $=$ Indice dei tipi lessicali e altre modalità di consultazione (2008). Scarmagno : Priuli \& Verlucca Editori [volume ; CD-Rom].

ALEPO III = Atlante Linguistico ed Etnografico del Piemonte occidentale - ALEPO. Volume III, Il mondo animale; Modulo I, Fauna selvatica; Modulo II Caccia e Pesca (2013). Alessandria : Ed. dell'Orso [volume, carte, CD-Rom].

ALI = Atlante Linguistico Italiano (1995 e sgg). Roma : Istituto Poligrafico e Zecca dello Stato.

ALiR = Atlas Linguistique Roman, Volume I (1996), Présentation, Commentaires ; Volume II.a (2001), Commentaires ; Volume II.b (2009), Commentaires, Roma : Istituto poligrafico e Zecca dello Stato, Libreria dello Stato.

ALJA = J.B. Martin - G. Tuaillon, Atlas Linguistique et Ethnographique du Jura et des Alpes du Nord (1971-1978). Paris : CNRS.

ALP = J.C. Bouvier - C. Martel, Atlas Linguistique et Ethnographique de la Provence (1975 e sgg). Paris : CNRS.

Baratto G. (2011). « Il trattamento degli etnotesti. Prime riflessioni e proposte per la realizzazione dei moduli di carattere 
Parole e testi

etnografico dell'ALEPO », Bollettino dell'Atlante Linguistico Italiano 35 : 43-77.

Bertoldi V. (1927). «Per la storia del lessico botanico popolare. A proposito di una recente pubblicazione », Archivum Romanicum XI/1 : 14-30.

Bouvier J.-C. (1992) «La notion d'ethnotexte », in J.-N- Pelen \& C. Martel (éd.) Les voies de la parole. Ethnotextes et littérature orale. Approches critiques. Aix-en-Provence: Publications de l'Université de Provence, 12-21.

Bouvier J.-C., Bremondy H. P., Joutard P., Mathieu G. \& Pelen J.-N. (1980). Tradition orale et identité culturelle. Problèmes et méthodes. Parigi : Ed. CNRS

Calleri D. (1990). «Messa a punto di un questionario per la raccolta di fitonimi dialettali », in G. Berruto \& A. Sobrero (éd.) Studi di sociolinguistica e dialettologia italiana offerti a Corrado Grassi, Galatina : Congedo, 77-94.

Calleri D., Canobbio S. \& Telmon T. (2001). «I fiori dell'ALEPO », in G.L. Beccaria \& C. Marello (éd.) La parola al testo. Scritti per Bice Mortara Garavelli. Alessandria : Edizioni dell'Orso, I, 95-118.

Canobbio S. (1985). «Testi dialettali ed etnotesti nell'atlante linguistico ed etnografico del Piemonte occidentale: appunti per una classificazione », in T. Telmon \& S. Canobbio (éd.), 207-343.

Canobbio S. (1989). « Al di là della raccolta dialettale : etnotesti e documentazione ergologica nell'ALEPO ", in AA.VV., Atlanti regionali : aspetti metodologici, linguistici e etnografici, Atti del XV Convegno del CSDI (Palermo 7-11 ottobre 1985). Pisa : Pacini, 83-106.

Canobbio S. (1999). «I saperi naturalistici e gli atlanti linguistici : l'esperienza dell'ALEPO », Quaderni di semantica XX/1 : 159-168.

Canobbio S. (2001). «Le rapport dialectique entre Passé, Présent et Futur dans les Ethnotextes de l'Atlas Linguistique et ethnographique du Piémont Occidental », in Le temps 


\section{S. CANOBBIO}

bricolé, n. monografico Le monde alpin et Rhodanien 3-4 : 189-202.

Canobbio S. (2002). «L'Atlante linguistico come strumento di ricerca ? A proposito di alcune esperienze italiane », in M.R. Simoni-Aurembou (éd.), 905-929.

Canobbio S. (2003). Le 'bestiole' dell'ALEPO, in R. Caprini (éd.) Parole romanze. Scritti per Michel Contini. Alessandria: Edizioni dell'Orso, 43-55.

Canobbio S. (2004). «Funghi e licheni nell'ALEPO », in ALEPO I-III : 39-54.

Canobbio S. (2005). «Alberi e arbusti nell'ALEPO », in ALEPO I-I : 45-60.

Canobbio S (2007). «Erbe e fiori nell'ALEPO », in ALEPO III : 45-57.

Canobbio S. (2011). «Etnotesti », in AA.VV. Per i linguisti del nuovo millennio. Scritti in onore di Giovanni Ruffino. Palermo : Sellerio, 114-117.

Canobbio S. (2012). «Elementi di ornitonimia piemontese : i rapaci notturni dell'ALEPO » in M. Oliviéri, G. BrunTrigaud \& P. Del Giudice (éd.) La leçon des dialectes. Hommages à Jean-Philippe Dalbera. Alessandria : Edizioni dell'Orso, 97-105 (con 3 tavole fuori testo).

Canobbio S. (2013). «Il mondo animale nell'ALEPO », in ALEPO III : 41-52.

Canobbio S. \& Raimondi G. (2004). «Fitonimi e fitotoponimi nel Piemonte alpino », in J.-C. Ranucci \& J.-P. Dalbera (éd.) Toponymie de l'espace alpin : regards croisés, Corpus. Les Cahiers 2 : 177-201.

Cerruti M. \& Regis R. (2007). «Language change and areal Linguistics : notes on Western Piedmont », Dialectologia et Geolinguistica $15: 23-43$

Cerruti M. \& Regis R. (2008). « La tipizzazione lessicale : problemi e metodi », in ALEPO I/Indice : 15-49.

Dalbera J.-P. (2002). " Géolinguistique : un nouveau souffle ? », in M.-R. Simoni-Aurembou (éd.), 831-849. 
Dalbera J.-P (2006). Des dialectes au langage. Une archéologie $d u$ sens. Paris : Honoré Champion Editeur.

Dalbera J.-P. (2009). "Quel avenir pour la dialectologie ?», in B. Horiot (éd.) La dialectologie hier et aujourd'hui (19062006). Lyon : Centre d'études linguistiques Jacques Goudet, 455-468.

Duberti N. \& Miola E. (2012). Alpi del mare tra lingue e letterature. Pluralità storica e ricerca di unità. Alesssandria : Edizioni dell'Orso.

Evans D. (1960). «Noms et folklore des oiseaux de proie nocturnes dans le Midi de la France », Revue de langue et littérature provençales $3: 5-25$.

Matranga V. \& Sottile R. (éd.) (2007). Esperienze geolinguistiche. Percorsi di ricerca italiani e europei, ALS, Materiali e ricerche 18, Palermo.

Mendicino A., Prantera N. \& Maddalon M. (éd.), (2004). Etnolinguistica e zoonimia. Le denominazioni popolari degli animali. Rende : Università della Calabria.

Ortalli G. \& Sanga G. (éd.), (2004). Nature Knowledge. Oxford : Berghahn

Prantera N., Mendicino A. \& Citraro C. (éd.), (2010). Parole. Il lessico come strumento per organizzare e trasmettere gli etnosaperi. Università della Calabria, Centro editoriale e librario, Volume con CD.

Raimondi G. (2003). «L'Atlante », in ALEPO/Presentazione : 53-97.

Regis R. (2001). «Il mistilinguismo nei materiali dell'ALEPO : un approccio funzionale », Bollettino dell'Atlante Linguistico Italiano $25: 67-80$.

Regis R. (2006). "Incontri, scontri, reazioni : il prestito nei materiali dell'ALEPO ", in Diglossie et interférences linguistiques: néologismes, emprunts, calques. Actes de la conférence annuelle sur l'activité scientifique du Centre d'Etudes francoprovençales. Aosta, 155-169. 


\section{S. CANOBBIO}

Regis R. (2008). «Il Tipo corylus : origine, riscontri, fortuna (con particolare riferimento al territorio italiano », Vox romanica $67: 11-33$.

Simoni-Aurembou M. R. (éd.), (2002). « Nouveaux regards sur la variation diatopique", Revue belge de Philologie et d'Histoire LXXX.

Telmon T. (2009). «La geografia linguistica: una scienza ambigua o una scienza duplice? », Rivista italiana di dialettologia $33: 17-25$.

Telmon T. \& Canobbio S. (éd.), (1985). Atlante Linguistico ed Etnografico del Piemonte occidentale. Materiali e Saggi 1984. Torino : Regione Piemonte.

Varotto M. (éd.), (2013). La montagna che torna a vivere. Testimonianze e progetti per la rinascita delle Terre Alte. Portogruaro : Nuova Dimensione. 